Supplement of Saf. Nucl. Waste Disposal, 1, 293-294, 2021

https://doi.org/10.5194/sand-1-293-2021-supplement

(c) Author(s) 2021. CC BY 4.0 License.

(c) (1)

Supplement of

\title{
Transport in tight material enlightened by process tomography
}

Johannes Kulenkampff et al.

Correspondence to: Johannes Kulenkampff (j.kulenkampff@hzdr.de)

The copyright of individual parts of the supplement might differ from the article licence. 


\section{Transport in tight material enlightened by process tomography}

Johannes Kulenkampff, Till Bollermann, Maria A. Cardenas Rivera, and Cornelius Fischer Institute of Resource Ecology - Reactive Transport, Helmholtz-Zentrum Dresden-Rossendorf contact: (j.kulenkampff@hzdr.de)
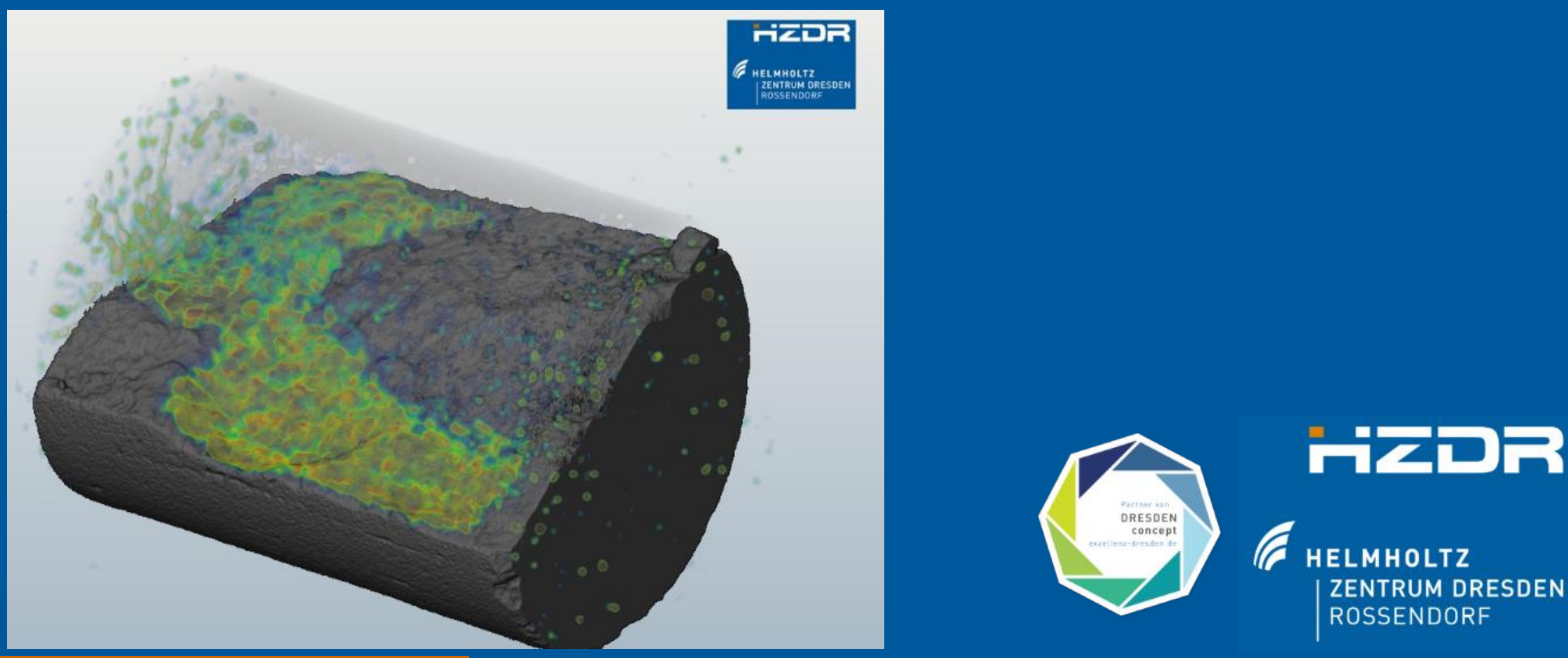

HELMHOLTZ

ZENTRUM DRESDEN ROSSENDORF 


\section{Relevance of laboratory results}

Reliable and robust data basis,

but discrepancies to large scale.

Questions to be asked:

Permeability frequently increases with scale, why?

Is the experimental time scale appropriate?

Do we understand the processes on the pore scale?

Impact of heterogeneity 


\section{Transport in tight materials}

Input-Output tests

(Permeability, diffusion cells, BTCs)
Process tomography

(PET- $\mu \mathrm{CT})$

\section{Duration of tests}

Stability?

Time expenses
Spatiotemporally resolved snapshots

Prove of stability by observation

Optimization of test procedure

Instationary tests without signal at outlet

Spatial Representativity

Intact samples preferred (biased selection of samples) Small samples preferred (plugs)
Applicabillity on disturbed samples - including heterogeneities and fractures Tomography on complete drill cores Information on REV size Identification of connected transport paths

Process understanding, modelling

Bias by test method

Identification of process (advection, diffusion, interactions with matrix) 


\section{Benefits of Process Tomography}

Illuminate spatiotemporal internal properties during the process

- Heterogeneity (e.g. preferential transport, reactive zones)

- Retention and storage

- Velocity or rate distributions

Downside:

- Experimental limitations

- Expensive (work and costs) 


\section{Principle of Process Tomography with GeoPET}
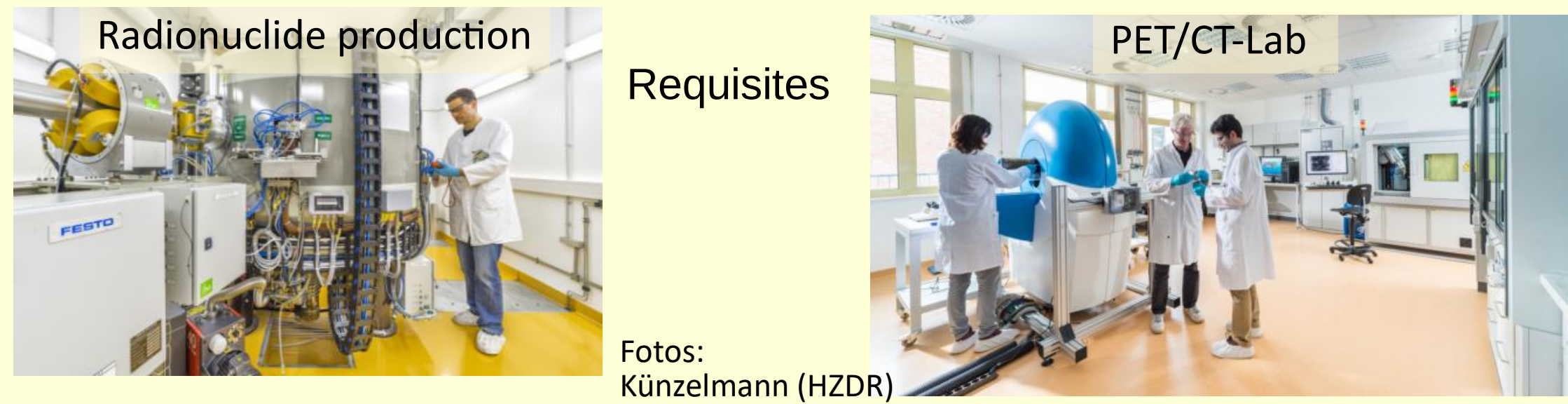

Plastic pressure vessel or cast in epoxy

Transparent for PET and CT $\max . p_{c}: 10$ bar

Sample dimensions: $\mathrm{d}: 30 . .100 \mathrm{~mm}$ l: $<100 \mathrm{~mm}$ Injection of tracer pulse into continuous flow of carrier solution. constant flow: $1 \mu \mathrm{L} / \mathrm{min} . .1 \mathrm{~mL} / \mathrm{min}$

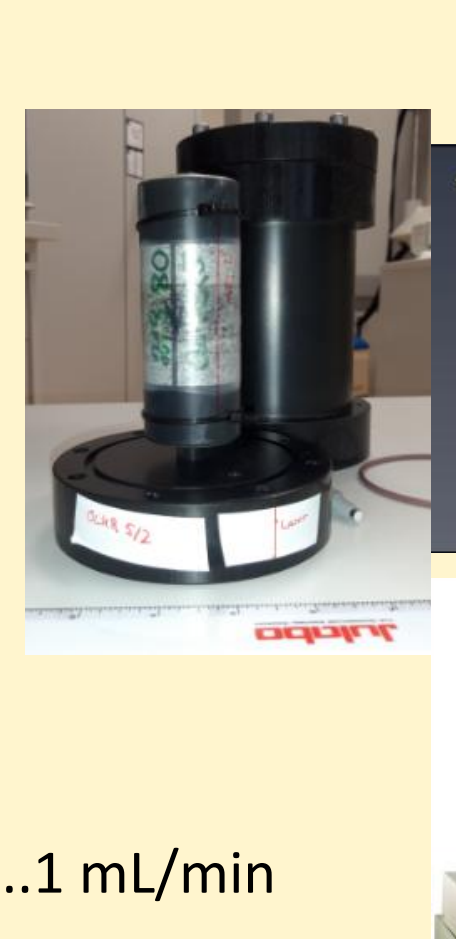

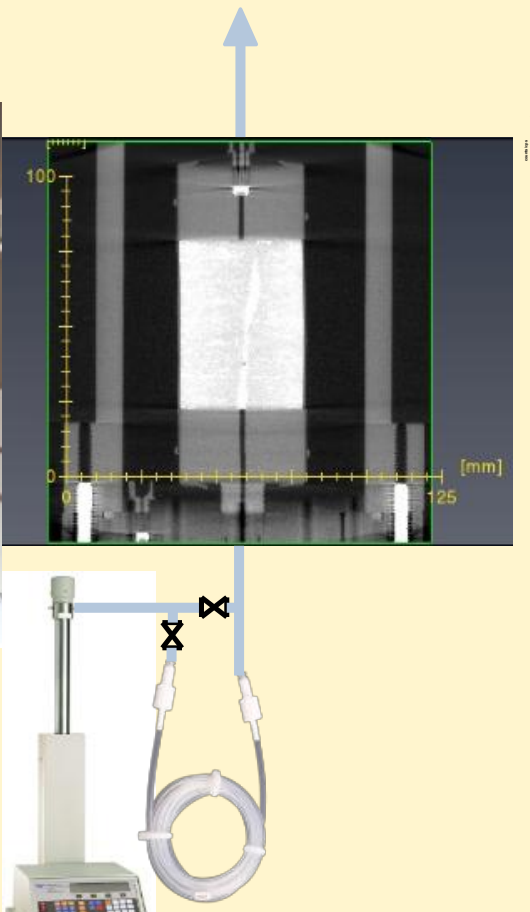

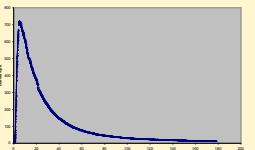

BTC from flow-through counter

Data: suite of PET frames (minimum frame rate $1 \mathrm{~min}$, acquisition time hours to months) plus $\mu \mathrm{CT}$ image tomographic reconstruction 4D image processing parameterization 


\section{Example for flow experiments}

Bukov granite with fracture

Flow rate: $0.1 \mathrm{~mL} / \mathrm{min}$

Carrier solution: $1 \mathrm{mMol} \mathrm{KF}$ Tracer: $1 \mathrm{~mL}\left[{ }^{18} \mathrm{~F}\right] \mathrm{KF}$
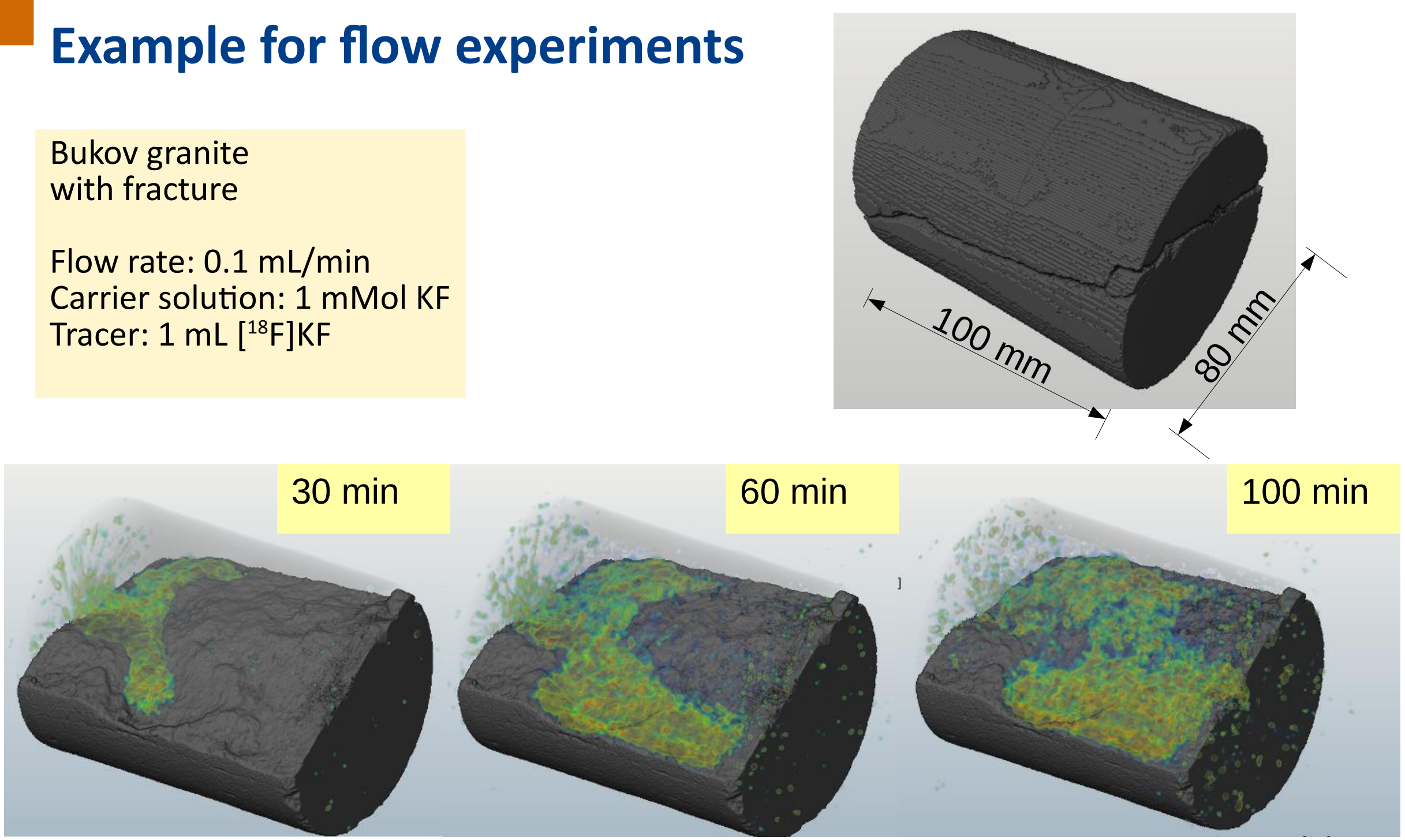

Kulenkampff, J.: Geophysical Research Abstracts, Vol. 20, EGU2018-8813-1, 2018. Fischer, C. et al.: Geophysical Research Abstracts, Vol. 21, EGU2019-13965, 2019. 


\section{Results from Flow Experiment}

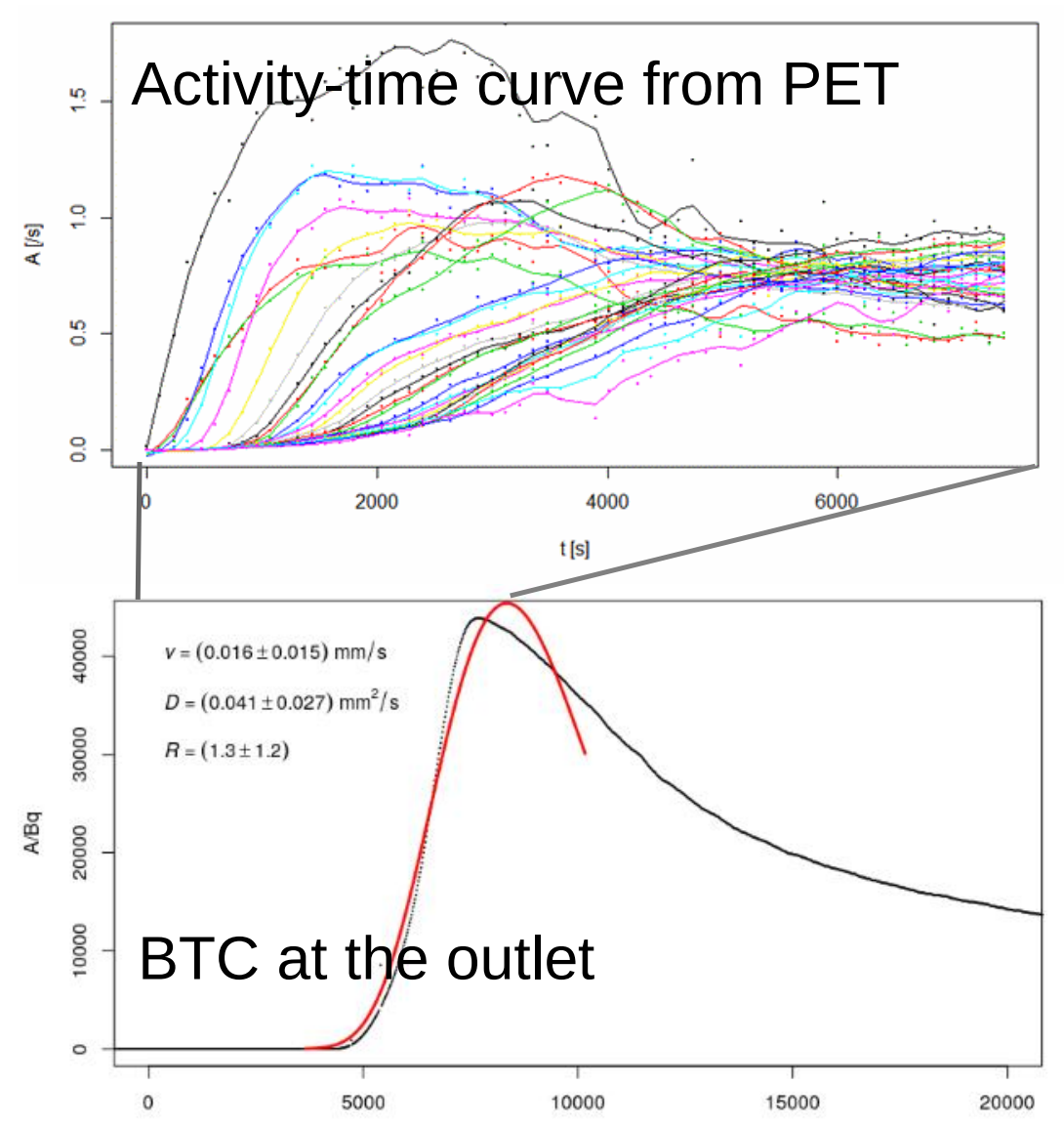

Internal and external BTCs

Small scale dispersion Information on retardation

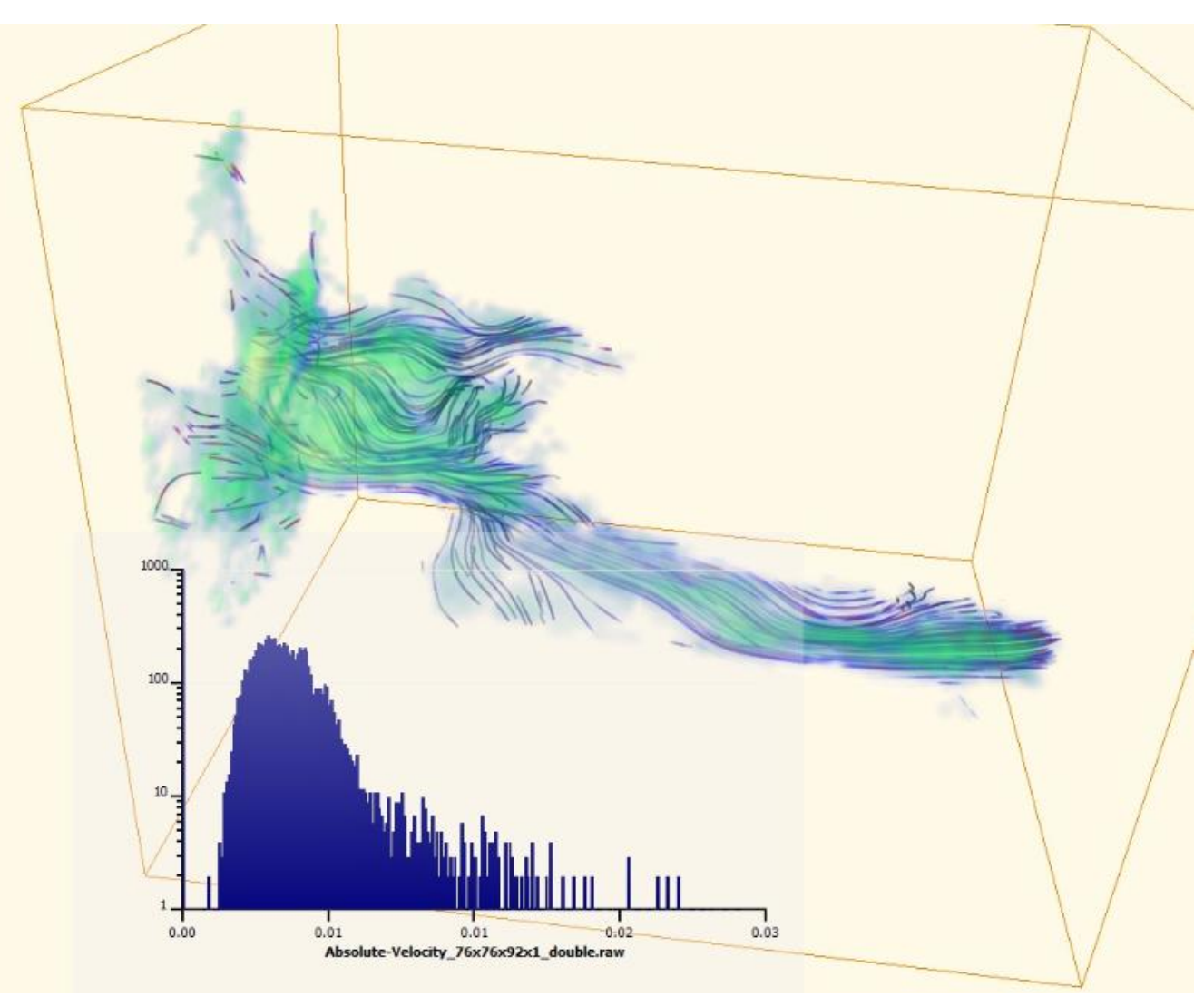

Flow path distribution Velocity histogram Effective volume 


\section{Examples for diffusion}

Opalinus clay

Completely cast in epoxy Axial drill hole filled with [22 Na] synthetic OPA-water 19 PET-frames over 1 year
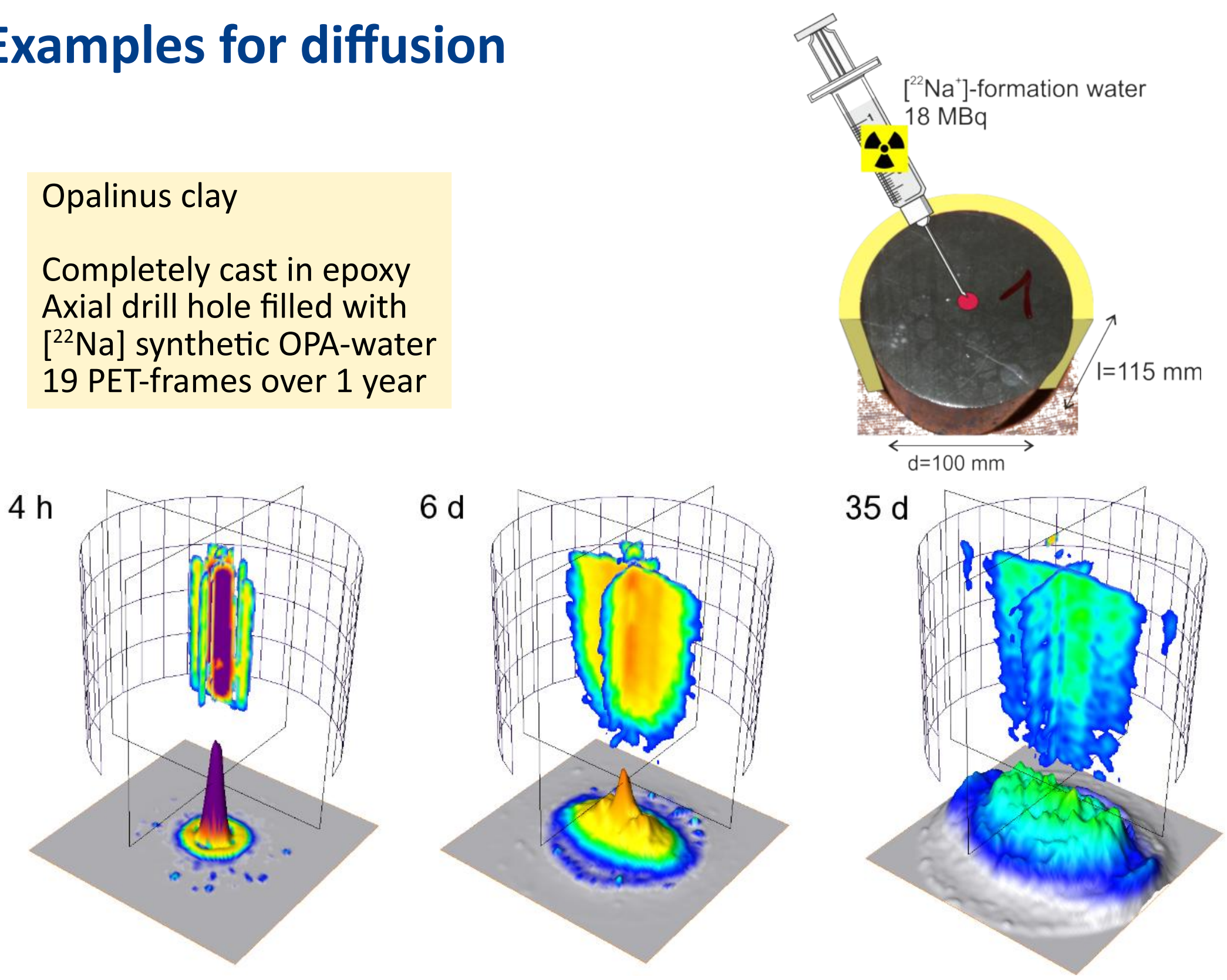

Kulenkampff, J. et al.: Solid Earth 7, 1207-1215, 2016. 


\section{Diffusion Results}

PET data

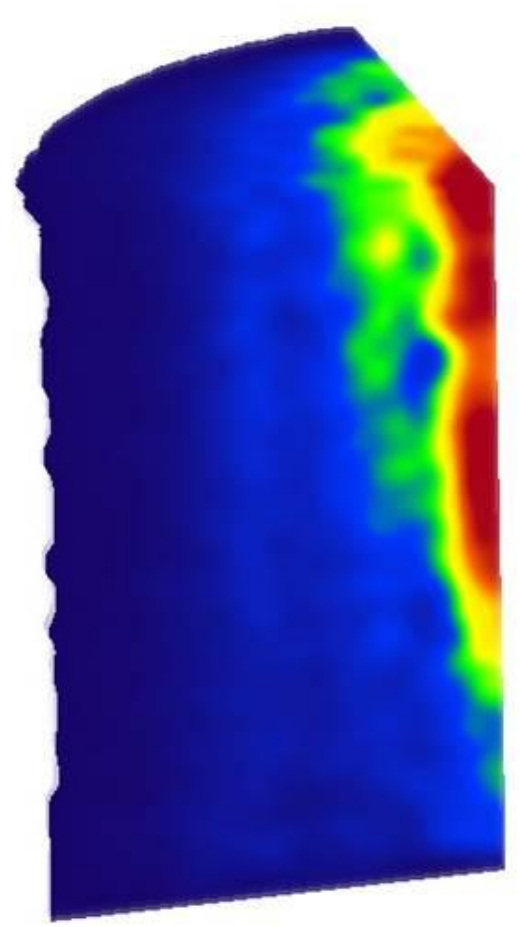

COMSOL best fit

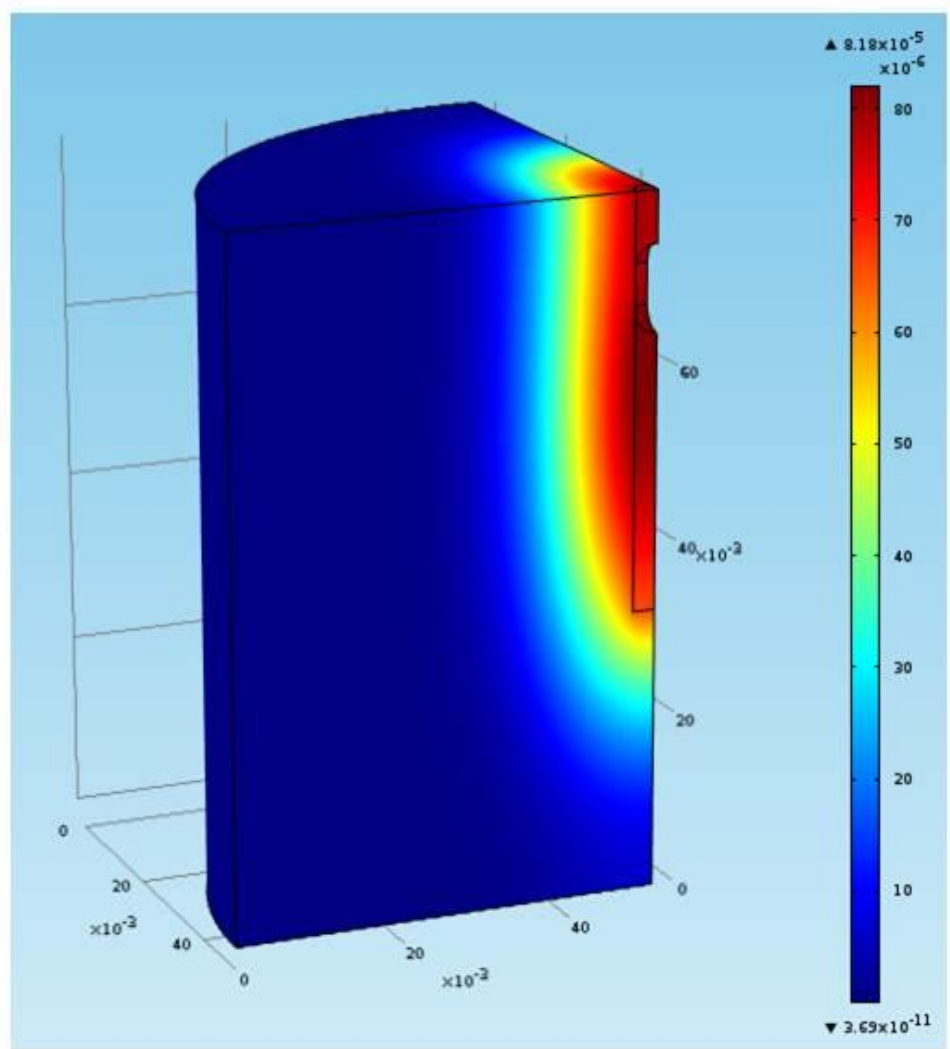

Best fit to FEM yields

Diffusion tensor

(axisymmetric) Anisotropy Heterogeneity

Lippmann-Pipke, J. et al.: Computers and Geosciences, 101, 21-27, 2017 


\section{Achievements}

- process identification and understanding

- images (affirmative or puzzling)

- permeability or diffusion coefficient (eventually as tensors)

- dispersivity

- internal propagation curve

- transport pathways

- velocity distribution

- effective volume

- heterogeneity

- scaling from $\mathrm{mm}$ to $\mathrm{cm}$

Questions

- applicability for safety case

- how to parameterize (e.g. heterogeneity parameter)

- how to apply for upscaling 


\section{Acknowledgements}

This research has received support by

Bundesministerium für Bildung und Forschung BMBF

and the Helmholtz Gemeinschaft (HGF)

(grant no. 02NUK053B)

Bundesministerium für Wirtschaft und Energie BMWi

(grant nos. 02E11748B and 02E11769B),

European Commission Horizon 2020

(grant nos. 662147 (Cebama) and 847593 (EURAD)) 